\title{
Methodology for Characterizing High-Risk Orbital Debris in the Geosynchronous Orbit Regime
}

\author{
Paul V. Anderson ${ }^{\mathrm{a}, 1, *}$, Hanspeter Schaub ${ }^{\mathrm{a}, 2}$ \\ ${ }^{a}$ University of Colorado, Boulder, CO, 80309, USA
}

\begin{abstract}
Forecasting of localized debris congestion in the geostationary (GEO) ring is performed to formulate and investigate methodology for identifying the debris objects that pose the highest risk to operational satellites in this ring. Proximity and speed relative to GEO during near-miss events detected under a torus intersection metric are translated into a combined risk factor that is accumulated during propagation. This accumulated risk is then used to identify the objects that have the highest risk contributions, either globally or in the vicinity of one of the two gravitational wells at $75^{\circ} \mathrm{E}$ and $105^{\circ} \mathrm{W}$. Results show that nearly $60 \%$ of the total risk surrounding the Western well is attributed to 10 derelicts alone, which has critical implications for active debris removal (ADR) target selection for attenuating risk levels in this ring.
\end{abstract}

Keywords: orbital debris, geosynchronous orbit, ADR target selection

\section{Introduction}

The geostationary (GEO) regime is a unique commodity of the terrestrial satellite industry that is becoming increasingly contaminated with orbital debris (Johnson, 1999; Jehn et al., 2005), but is heavily populated with high-value assets (Chrystal et al., 2011). As the lack of atmospheric drag effects at the GEO altitude renders lifetimes of these debris essentially infinitely long, conjunction assessment must be performed to safeguard operational GEO satellites from potential collisions with the uncontrolled derelict field. GEO satellites must maintain a specified longitude slot, and cannot simply shift in phase to evade debris. Therefore, studies of the macroscopic behavior of the GEO debris population are required to describe debris fluxes through particular GEO longitude slots, and forecast how frequently operational assets in these regions must potentially perform maneuvers to mitigate conjunctions. Rather than presenting the high-precision analysis demanded by risk assessment and mitigation measures, this study builds upon the methods of Anderson and Schaub (2013),

\footnotetext{
*Corresponding author

Email addresses: paul.anderson@colorado.edu (Paul V. Anderson), hanspeter.schaub@colorado.edu (Hanspeter Schaub)

${ }^{1}$ Graduate Research Assistant, Department of Aerospace Engineering Sciences, 429 UCB, Boulder, CO, 80309 .

${ }^{2}$ Professor, Department of Aerospace Engineering Sciences, 429 UCB, Boulder, CO, 80309.
} 
which illustrates a one-year, macroscopic congestion forecast for debris at GEO, to determine which localized regions of the GEO ring are, in general, most susceptible to rising levels of debris congestion. As overcrowding of this ring is growing into a serious concern for owners and operators internationally, knowledge of debris flux patterns - termed debris weather - is critical for space situational awareness activities at GEO. Of a significant interest is determining which classes of uncontrolled derelicts contribute the most to congestion and risk levels, both globally and locally, over a specified time frame. Assessing if subsets of the debris population contribute homogeneously to congestion and risk levels across GEO is imperative information for active debris removal (ADR) initiatives seeking to attenuate localized risk in particular longitude slots to sustain slot utilization.

Existing debris analysis tools (Lewis et al., 2001; Klinkrad, 2006) rely upon inertially-fixed cells to detect debris cell passage events (CPE), such that densities are often averaged over cell right ascension, and provided as a function of altitude and declination (Klinkrad, 2006). Following Anderson and Schaub (2013), this study harnesses a toroidal cell configuration in the GEO ring to investigate the impact of various classes of large-scale, uncontrolled objects on congestion and risk levels from a longitude-dependent perspective. Small-scale debris sources such as explosion and collision events, although considered recently in Hansen and Sorge (2013) and Oltrogge and Finkleman (2008), are not considered in this study, nor are other growth mechanisms such as ejection of solid rocket motor (SRM) slag or shedding of multi-layered insulation (MLI) (Wegener et al., 2004).

Using publicly-available U.S. Strategic Command tracking data and an orbit classification system established by the European Space Agency (Flegel et al., 2009; Flohrer, 2014), this paper will illustrate a five-year debris forecast in the GEO ring for large-scale, trackable, and unclassified resident space objects (RSOs) with up-to-date two-line element (TLE) sets. The results of this five-year debris simulation - which performs parallel propagation using $4 \times 4$ EGM-96 gravitation, luni-solar perturbations, and a nominal solar radiation pressure effect - are used to characterize which classes of uncontrolled debris objects contribute the most to longitude-dependent risk levels, globally and over a defined subset of longitude slots. In particular, methodology for identifying the objects that contribute the largest percentage to the total risk level accumulated either globally or locally during the forecasting period is presented. For ADR initiatives geared towards slot clean-up at particular longitudes, information such as that determined from debris simulations harnessing the torus intersection metric to evaluate longitude-dependent risk is especially useful in helping to determine which individual objects should be removed to maximize reduction in risk, either globally or across a defined subset of longitude slots, namely, in the vicinity of the high-impact and debriscritical gravitational wells positioned at $75^{\circ} \mathrm{E}$ and $105^{\circ} \mathrm{W}$ (Anderson and Schaub, 2013).

The results of this work may thus be harnessed in tandem with long-term debris prediction studies, such as that performed in Anderson and Schaub (2014), to provide recommendations for architecture and design of potential ADR demonstration missions in the GEO regime. Studies led by the NASA Orbital Debris Program Office use the product of a debris object's mass and its probability of collision at a desired epoch as the ADR target selection criterion for identifying the top ADR targets in the LEO regime (Liou, 2011). ADR target selection studies for the GEO regime have received less attention than equivalent LEO ADR target studies (Liou, 2011; Peterson, 2012; Quinlan et al., 2011). This study seeks to begin filling this void in the literature by formulating methodology for identifying the top ADR targets at 
Table 1: Orbit categories applied to geosynchronous objects extracted from 02/28/2014 TLE set.

\begin{tabular}{c|c|l} 
Class & Type & \multicolumn{1}{|c}{ Description } \\
\hline C1 & Controlled & Longitude/inclination control (E-W/N-S control) \\
C2 & Controlled & Longitude control only (E-W control only) \\
D & Drifting & Circulating above/below/through GEO altitude \\
L1 & Librating & Libration about Eastern stable point at $75^{\circ} \mathrm{E}$ \\
L2 & Librating & Libration about Western stable point at $105^{\circ} \mathrm{W}$ \\
L3 & Librating & Libration about Eastern and Western stable points \\
IN & Indeterminate & Unknown status (e.g., recent TLE not available)
\end{tabular}

GEO, based on the torus intersection metric and independent of the statistical probability of collision measure often used in conjunction assessment, a computation that requires position covariance information. Note that this torus intersection metric - which is useful for gauging localized congestion at GEO - does not claim that the identified ADR targets might actually collide with specific operational satellites at GEO. The source of orbital data for this study is the publicly-available TLE sets, which are not accurate enough to forecast conjunction events over the long-term, and do not include covariance information. Rather, instead of evaluating satellite-specific probabilities of collision against the large-scale debris population, this study seeks to identify the specific GEO debris objects in this population that contribute the highest levels of macroscopic risk to the GEO regime in general, both globally and in the vicinity of the gravitational wells. In this sense, potential conjunction events with high-macroscopic-risk objects are more threatening in that these derelicts are routinely passing through particular longitude slots at GEO with non-trivial, potentially catastrophic velocities relative to GEO.

\section{Current RSO Population at GEO}

The RSO population in the GEO regime is categorized using the orbit taxonomy applied in the European Space Agency's DISCOS database (Database and Information System Characterising Objects in Space) (Flohrer et al., 2013). For GEO objects, seven categories are selected to classify the types of orbits exhibited - two controlled classes and five uncontrolled classes. Note that only the uncontrolled objects are assumed to contribute to localized debris congestion in this study. GEO RSOs are selected according to the orbit element bounds used in the European Space Agency's Classification of Geosynchronous Objects reports (Flohrer, 2014): eccentricity less than $0.2(e<0.2)$, inclination less than $70^{\circ}\left(i<70^{\circ}\right)$, and mean motion between 0.9 and 1.1 revolutions per sidereal day $(0.9<n<1.1)$, corresponding to the semi-major axis range $-2596 \mathrm{~km}<a-a_{\mathrm{GEO}}<3068 \mathrm{~km}$ relative to GEO. Per these element bounds, launch vehicle upper stages are only included in the congestion forecast if they exhibit GEO-like orbits. As a result, rocket bodies in highly-eccentric geostationary transfer orbits (GTOs) are not considered, even though these trajectories are closely approaching — or crossing through - the GEO altitude at or near apogee.

Orbital data is obtained from the publicly-available two-line element (TLE) sets provided 


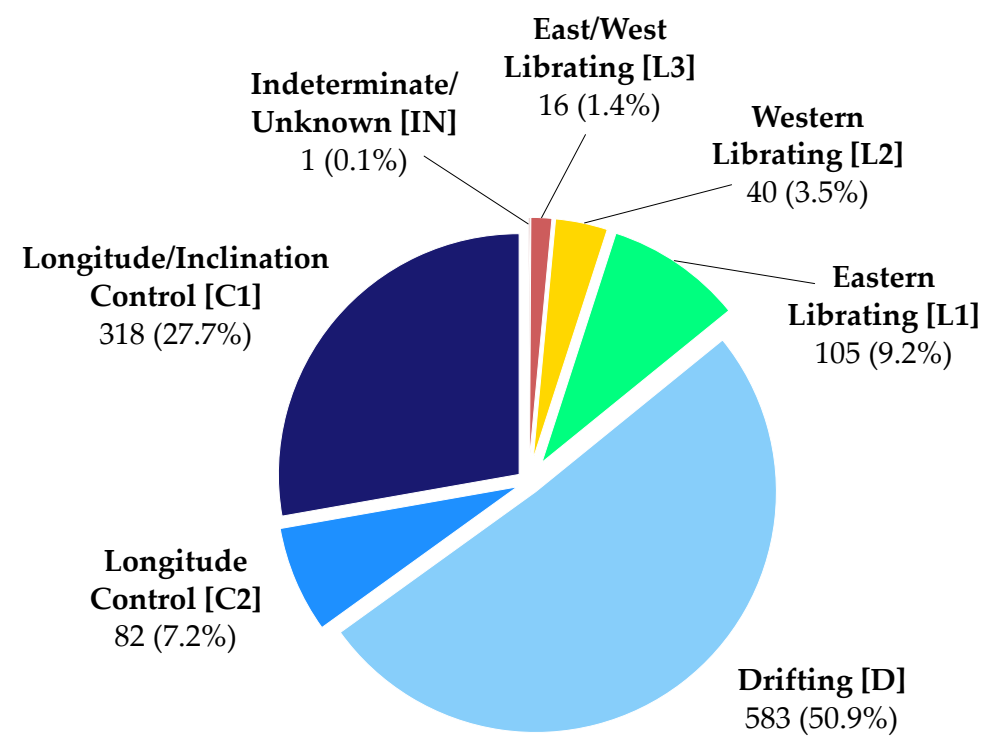

Figure 1: GEO orbit class distribution for 02/28/2014 TLE set.

by U.S. Strategic Command (USSTRATCOM). ${ }^{3}$ For this study, a reference TLE set obtained on February 28, 2014 is employed; the class distribution for the 1145 objects extracted from this set is shown in Figure 1. TLE data are provided in the form of doubly-averaged Keplerian elements with mean motion instead of semi-major axis (Klinkrad, 2006), transformed into Cartesian states in the true equator, mean equinox (TEME) frame (Vallado, 2007) with SGP-4 theory (Hoots and Roehrich, 1980). ${ }^{4}$ Note that because of the limited accuracy of TLE sets, these data are not intended for studies that require highly-precise orbit prediction capabilities. As the purpose of this paper is to identify "high-risk" debris objects via localized congestion metrics on a macroscopic scale - not on a specific, satellite-to-satellite basis - the accuracy of these data is sufficient. ${ }^{5}$ Furthermore, as only objects larger than approximately 0.8-1.0 meter in effective diameter are actively tracked at the GEO altitude (Flohrer, 2014), only objects at least of this size are considered here. Since this study only incorporates the trackable, catalogued, and unclassified GEO population with recent TLE sets, the findings of this study serve to illustrate a lower bound of the actual debris congestion and risk situation at GEO. This congestion and risk information is then harnessed to identify which large-scale objects at GEO contribute the most to risk levels across this arena, both globally and in the vicinity of the two gravitational wells.

Furthermore, as risk to operational satellites at GEO is the core focus of this study, debrisdebris collision risk among the 745 uncontrolled objects in Figure 1 is not treated. This type of collision risk - which can be mitigated strictly by ADR since no maneuverable objects are involved - is another dimension of the debris challenge at GEO that should be considered in

\footnotetext{
${ }^{3}$ Publicly-available TLE data sets are available for bulk download from https://www.space-track.org/

${ }^{4}$ ANSI-C implementation of merged SGP-4/SDP-4 theory is available from http://www. sat.dundee . ac.uk/ psc/sgp4.html (Vallado et al., 2006)

${ }^{5}$ Numerical justification for using TLE data sets in a similar GEO debris congestion study is provided in Anderson and Schaub (2014).
} 
a dedicated paper addressing this concern. It is therefore important to note that the highestrisk objects identified in this study are not necessarily those that exhibit the highest debrisdebris collision potential, which could be accounted for by additional terms in the risk metric introduced in Section 3.2.

The Eastern librating (L1) and Western librating (L2) objects oscillate within the Earthfixed frame around their respective gravitational wells with a libration period of 816 days at minimum for small amplitudes (Klinkrad, 2006). Using tabulated data in Flohrer (2014) in tandem with the analytic libration theory from Allan (1963), a histogram of the libration periods for the $145 \mathrm{~L} 1 / \mathrm{L} 2$ objects included in this study is shown in Figure 2. The median of this libration period distribution is approximately 2.6 years, such that a five-year time frame is used for this study to capture two mean cycles of the libration motion. In this manner, any bias introduced in global and local risk results towards a particular orbit class is minimized.

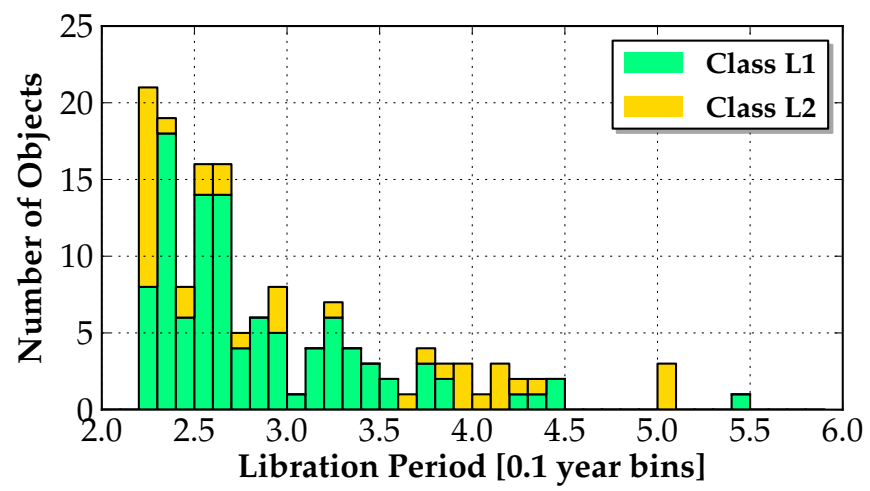

Figure 2: Libration periods for L1/L2 objects included in study.

\section{Methodology for Forecasting Localized GEO Risk}

\subsection{Overview of Near-Miss Events Metric}

The following metric for quantifying longitude-dependent orbit debris congestion at GEO is discussed in detail in Anderson and Schaub (2013), and is summarized in this section for convenience. "Near-miss events" for the GEO longitude slots are determined by formulating a GEO-encompassing torus with major radius $r_{\mathrm{GEO}}=42164 \mathrm{~km}$ and minor radius $\tilde{r}$, partitioned into longitude increments of $\Delta \lambda=1.0^{\circ}$ (Anderson and Schaub, 2013). The minor radius $\tilde{r}$ is equivalent to the radius of the circular torus cross-section, and provides a means to evaluate debris congestion levels occurring within various distances of the GEO longitude slots - a larger minor radius captures more near-miss events. For this study, a representative minor radius $\tilde{r}=50 \mathrm{~km}$ is considered, as this proximity to GEO is a rough upper bound for distances at which conjunction assessment could be considered for operational GEO assets. ${ }^{6}$

\footnotetext{
${ }^{6}$ The terminology "near-miss event at $50 \mathrm{~km}$ " could also be interpreted as a sub-50 km conjunction event. It is important to emphasize, however, that the near-miss event metric is a measure of localized congestion only, and is independent of the probability of collision or other risk metrics applied in operational conjunction scenarios. Thus, the "risk" functions introduced in Section 3.2 are intended to be for heuristic purposes only.
} 
Further, this torus formulation is a natural choice for evaluating near-miss events for these non-inertial GEO slots, as torus geometry is invariant as seen by both Earth-centered inertial frame (i.e., J2000) and Earth-centered, Earth-fixed frame, in which these GEO longitude slots are stationary (Anderson and Schaub, 2013).

Near-miss events are detected during propagation of an object by checking for the transversal of this GEO torus boundary at each time step during numerical integration; if finer resolution is desired, an interpolation algorithm can be employed to check for torus intersections between integration time steps. Mathematically, a near-miss event occurs if (Anderson and Schaub, 2013)

$$
\left(r_{\mathrm{GEO}}-\sqrt{r_{X}^{2}+r_{Y}^{2}}\right)^{2}+r_{Z}^{2}-\tilde{r}^{2}<0
$$

is satisfied, where $\left(r_{X}, r_{Y}, r_{Z}\right)^{T}$ is the RSO position vector expressed in inertial frame components. The longitude of intersection $\lambda_{\mathrm{CPE}}$ is thus determined as:

$$
\lambda_{\mathrm{CPE}}=\arctan \left(\frac{r_{Y}}{r_{X}}\right)-\alpha_{G}
$$

where $\alpha_{G}$ is the right ascension of Greenwich (Greenwich sidereal time) (Curtis, 2005). When a torus-intersection is detected with Equation (1), the longitude of intersection is determined with Equation (2), and the total near-miss count for the corresponding cell is updated. To ensure that equivalent intersections are not accounted for more than once during near-miss event detection, counting logic is called before a cell intersection counter is updated to screen the event for redundancy. The full algorithm for quantifying congestion via near-miss events with the GEO torus formulation is detailed in Anderson and Schaub (2013).

\subsection{Risk Functions for GEO-Relative Position and Velocity}

To begin identifying the particular uncontrolled objects responsible for the highest levels of both global and localized risk in the GEO regime, the GEO-relative position and velocity for an object at torus intersection must be translated into a risk metric that can be applied to categorize and rank the GEO debris population based upon levels of globally- and locallycontributed risk. It is important to note that the number of near-miss events for each object over the duration of the forecasting period is an insufficient measure for evaluating both global and localized risk, since the proximity of these near-miss events to the GEO altitudeand the GEO-relative velocity with which these near-misses occur - are not explicitly taken into account. For this study, each near-miss event is assigned a level of combined risk that is based on the GEO-relative state of the responsible debris object at the time of torus intersection. Position and velocity risk factor functions are employed to individually weight (a) how close the object comes to the GEO altitude, and (b) how fast the object is translating relative to the local GEO orbit velocity vector. Specifically, the position and velocity risk functions selected for this study are as follows:

$$
\begin{aligned}
& R_{r}(r)=\left(\frac{\tilde{r}-r}{\tilde{r}}\right)^{2}, \quad 0 \leqslant r \leqslant \tilde{r} \\
& R_{v}(v)=1-e^{-3 v / \bar{v}}, \quad v \geqslant 0
\end{aligned}
$$


The position risk factor function in Equation (3) is of quadratic form, rising smoothly from $R_{r}(\tilde{r})=0$ at the torus boundary to $R_{r}(0)=1$ at the GEO altitude. The velocity risk function in Equation (4) is of an exponential form, selected to rapidly saturate to $R_{v}(v) \rightarrow 1$ when the GEO-relative velocity surpasses a defined threshold, beyond which collision velocities are considered catastrophic. In this manner, all relative velocities beyond this critical threshold are weighted nearly equally - collisions with uncontrolled objects traveling with catastrophic relative speeds have serious and likely mission-ending consequences, regardless of where these relative speeds lie above the catastrophic threshold. The position and velocity risk factor functions defined in Equations (3)-(4) are illustrated for reference in Figure 3.

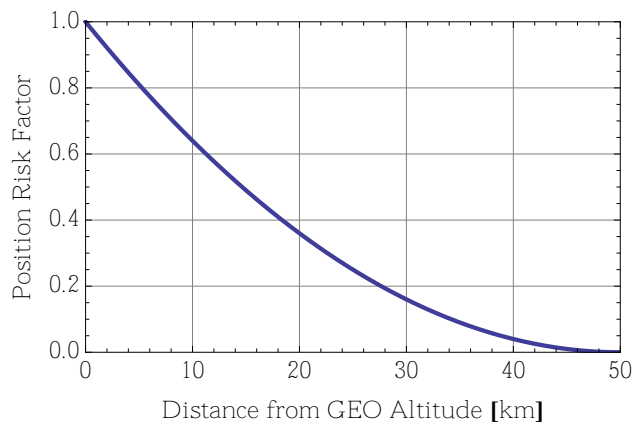

(a) Position risk function in Equation (3).

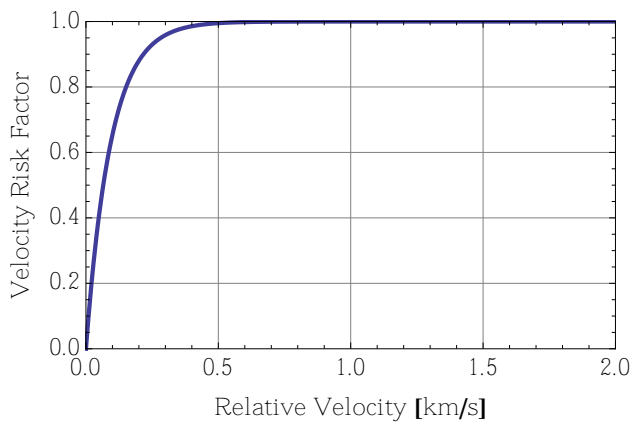

(b) Velocity risk function in Equation (4).

Figure 3: Functions selected for computing combined risk factor of each near-miss event.

The GEO-relative position $r$ is given by

$$
r=\sqrt{\left(r_{\mathrm{GEO}}-\sqrt{r_{X}^{2}+r_{Y}^{2}}\right)^{2}+r_{Z}^{2}}
$$

The GEO-relative velocity $v$ is computed by differencing the object's inertial velocity vector at torus intersection with the local, two-body orbit velocity vector at the longitude of intersection, expressed in Earth-centered inertial frame (i.e., J2000) components as

$$
\boldsymbol{v}_{\mathrm{GEO}}=\left[-\sqrt{\frac{\mu_{\oplus}}{r_{\mathrm{GEO}}}} \sin \left(\lambda_{\mathrm{CPE}}+\alpha_{G}\right), \sqrt{\frac{\mu_{\oplus}}{r_{\mathrm{GEO}}}} \cos \left(\lambda_{\mathrm{CPE}}+\alpha_{G}\right), 0\right]^{T}
$$

The GEO-relative velocity is scaled by the time-constant-like parameter $\bar{v}$ in the velocity risk function in Equation (4), such that when $v=\bar{v}$, the risk function $R_{v}(\bar{v})=1-e^{-3} \approx 0.950$ and begins saturating. ${ }^{7}$ For this analysis, the parameter $\bar{v}$ is derived from the NASA Standard Breakup Model (Johnson et al., 2001), which was developed for NASA's long-term debris environment software EVOLVE 4.0, and has been validated against catalogued debris clouds and ground-based experimental results for particles larger than $1 \mathrm{~mm}$ (Klinkrad, 2006). In particular, $\bar{v}$ is selected as the relative speed threshold above which on-orbit collisions become

\footnotetext{
${ }^{7}$ More generally, the parameter $n$ in an analogous function of the form $1-e^{-n v / \bar{v}}$ can be selected to adjust the value of the velocity risk factor at $v=\bar{v}$ explicitly, i.e., for a desired velocity threshold risk $R_{v}(\bar{v})$, then $n=-\ln \left(1-R_{v}(\bar{v})\right)$.
} 
"catastrophic" (complete disintegration of both objects), assuming equal masses $m_{t}$ and $m_{p}$ for the target and impactor objects in the collision event, respectively (Johnson et al., 2001):

$$
\tilde{E}_{p}^{*}=\frac{1}{2}\left(\frac{m_{p}}{m_{t}}\right) \bar{v}^{2} \rightarrow \bar{v}=\sqrt{\frac{2 \tilde{E}_{p}^{*} m_{t}}{m_{p}}} \approx 0.283 \mathrm{~km} / \mathrm{s}
$$

where $\tilde{E}_{p}^{*} \equiv 40 \mathrm{~kJ} / \mathrm{kg}$ is the specific energy threshold for a catastrophic collision (Klinkrad, 2006). Hanada et al. (2005) compare the predictions of the NASA Standard Breakup Model against low-velocity impact experiments performed at a velocity range less than $0.3 \mathrm{~km} / \mathrm{s}$, and conclude that the hypervelocity collision model in the NASA Standard Breakup Model can be applied to lower-velocity collisions, with minor modifications that do not affect Equation (7). An alternate catastrophic collision threshold introduced by McKnight (1991) is $10 \bar{v}^{2} m_{p} \geqslant m_{t}$ on a qualitative basis - applying $m_{p}=m_{t}$ as before, $\bar{v} \approx 0.316 \mathrm{~km} / \mathrm{s}$, close to the catastrophic collision threshold of $0.283 \mathrm{~km} / \mathrm{s}$ in Equation (7) per the NASA Standard Breakup Model.

After the GEO-relative position and velocity for the near-miss event are computed, the corresponding risk factor functions defined via Equations (3)-(4) are evaluated, such that the combined risk factor given by the product $R_{r}(r) R_{v}(v)$ is evaluated. This combined risk factor for the near-miss event is on the interval $[0,1]$ and provides a metric for gauging how "threatening" the near-miss is for a hypothetical GEO satellite stationed on the longitude of intersection. Under this metric, uncontrolled objects that closely pass the GEO altitudeand have catastrophic-collision-inducing relative speeds when doing so - will, in general, have a higher combined risk for near-miss events detected during propagation. Note that both close proximity and sufficient relative speed must be present in order for a near-miss event to be considered high-risk under this metric, e.g., higher-speed events near the torus boundary and lower-speed events closer to the GEO altitude are both de-weighted in the combined risk factor.

It is important to note that debris object mass is not considered in this combined risk factor, as it is used in the NASA Orbital Debris Program Office's studies to identify the top active debris removal (ADR) targets for the LEO regime (Liou, 2011). Mass information is not included within the publicly-available TLE data source harnessed in this study. If approximate mass information is available, an auxiliary mass function can be used to weight the combined risk factor, or each object's mass can be in-built by using a kinetic energy of the form $\frac{1}{2} m v^{2}$ in Equation (4) directly, i.e., by replacing relative speed risk with a kinetic energy risk. With these modifications, the combined risk for more massive objects will be emphasized, in a similar manner that the probabilities of collision for LEO objects are scaled by object mass in (Liou, 2011).

\subsection{Propagator and Implementation}

A special perturbations propagation routine implemented in ANSI-C and parallelized with OpenCL is implemented to propagate the uncontrolled RSO population forward in time and determine torus intersection events. ${ }^{8}$ A lower-fidelity, representative force model of the GEO environment is used for the added benefit of dramatically-decreased simulation run times.

\footnotetext{
${ }^{8}$ The OpenCL 1.2 Specification is available at: http://www.khronos.org/registry/cl/.
} 
Here, the two-body equations of motion are numerically integrated under $4 \times 4$ EGM-96 gravitation, luni-solar perturbations, and solar radiation pressure (SRP), modeled with the cannonball assumption described in (Vallado, 2007), and attenuated with the occultation algorithm given in (Montenbruck and Gill, 2000). The equations of motion are

$$
\ddot{\boldsymbol{r}}=-\frac{\mu_{\oplus}}{r^{3}} \boldsymbol{r}+\boldsymbol{a}_{\oplus}+\boldsymbol{a}_{\overparen{C}}+\boldsymbol{a}_{\odot}+\boldsymbol{a}_{\mathrm{SRP}}
$$

where the first term denotes two-body acceleration, $\boldsymbol{a}_{\oplus}$ is the acceleration due to the nonsphericity of Earth, $\boldsymbol{a}_{\varangle}$ and $\boldsymbol{a}_{\odot}$ are the third-body perturbations from the Moon and Sun, respectively, and $\boldsymbol{a}_{\mathrm{SRP}}$ is the SRP acceleration. SRP is modeled using the inverse-square diffusion formulation of the solar luminosity $L_{\odot} \approx 3.839 \times 10^{26} \mathrm{~J} / \mathrm{s}$, with coefficient of reflectivity $c_{r} \equiv 1.5$ and GEO-representative area-to-mass ratio $A_{\odot} / m=0.04 \mathrm{~m}^{2} / \mathrm{kg} .{ }^{9}$ This GEO force model is in agreement with the results by Hansen and Sorge (2013), which ranks the importance of incorporating various environmental perturbations in GEO force models for debris analysis over time scales ranging from 1 week to 10 years.

In higher-fidelity force models, coordinate transformations between Earth-fixed and Earthinertial frames use high-accuracy Earth orientation parameters to account for precession, nutation, and polar motion; software suites such as the SPICE toolkit can be harnessed to perform these complex coordinate transformations. ${ }^{10}$ In this parallel propagator, however, a lower-fidelity transformation that accounts strictly for $z$-axis rotation by Greenwich sidereal time is used for increased speeds at run time. Further, instead of extracting Moon and Sun vectors from the DE-421 ephemerides, this routine uses lower-precision formulas for the geocentric position of these bodies, as given in the 2013 Astronomical Almanac (USNO and UKHO, 2013). Anderson and Schaub (2014) provides verification of this lower-fidelity force model by comparing localized congestion results over a five-year period with those obtained under higher-fidelity forcing.

The propagator utilizes an eighth-order, predictor-corrector Gauss-Jackson integrator (Berry and Healy, 2004) initialized with the Prince-Dormand 8(7) algorithm for integration of the equations of motion in Equation (8). During initial propagation of the uncontrolled population to the forecast start date, and during near-miss event detection over the prediction span, a 10-minute time step is specified for sufficient fidelity in capturing near-miss events. To increase resolution without significantly increasing simulation run times, a Lagrange interpolation method is used to detect torus intersections in one minute increments between primary time steps.

\section{Results of Five-Year Localized GEO Risk Forecast}

As a precursor to identifying which classes of objects contribute the most to both global and localized risk levels in this regime, a five-year macroscopic congestion forecast is performed with the minor radius $\tilde{r}=50 \mathrm{~km}$, using the baseline RSO population in the $02 / 28 / 2014$

\footnotetext{
${ }^{9}$ (Schaub and Jasper, 2011) indicates that this ratio is representative for operational and defunct satellites at GEO; this value is thus used in the SRP computation for all uncontrolled objects considered in this study.

${ }^{10}$ Jet Propulsion Laboratory's SPICE toolkits are available at http://naif.jpl.nasa.gov/naif/ toolkit.html.
} 
TLE set. Controlled assets $(\mathrm{C} 1 / \mathrm{C} 2)$ are assumed to maintain their specified longitude slots, while the 745 uncontrolled objects extracted from this set are propagated forward in time and incorporated in the near-miss study. Note that this simulation is only assessing the congestion generated by the current debris population over a five-year time frame. Nominal population growth, ${ }^{11}$ fragmentation events, solid rocket motor (SRM) slag, multi-layered insulation (MLI) shedding, and other debris growth mechanisms considered by Wegener et al. (2004), for example, are not treated in this simulation. Again, the objective for this paper is to investigate which classes of large-scale, uncontrolled derelicts currently at GEO contribute the most to global and local risk levels in this regime, and determine the highest-risk subsets of these classes.

\subsection{Localized Congestion, Velocity, and Risk Results}

The "debris weather forecast" at GEO for the five-year analysis period is shown in Figure 4(a), which illustrates the number of near-miss events per day at $50 \mathrm{~km}$ for each of the longitude slots at GEO. Accumulation of uncontrolled objects around the gravitational wells at $75^{\circ} \mathrm{E}$ and $105^{\circ} \mathrm{W}$ is a well-known result, as is discussed by Chobotov (2002) and Luu and Sabol (1998), for example. This is a particularly troublesome notion, as operational GEO assets are typically inserted into longitude slots near these debris-critical longitudes (Anderson and Schaub, 2014), and the probability of collision in the vicinity of these gravitational wells is seven times larger than in surrounding regions at GEO (Chrystal et al., 2011; McKnight and Di Pentino, 2013). From Figure 4(a), controlled GEO satellites in the longitude slots neighboring the two gravitational wells are subject to $4-8$ sub-50 km conjunctions per day - a factor of four increase over less congested longitudes (e.g., Atlantic and Pacific Oceans), which experience a maximum of 1-2 near-misses per day at $50 \mathrm{~km}$. Note that the number of near-miss events during the forecasting period for any given longitude slot does not equate to the probability of collision used in operational conjunction assessment. For discussions as to the impact of the debris population on longitude-dependent probability of collision at GEO, see McKnight and Di Pentino (2013).

It is interesting to highlight that although the number of near-miss events for a particular longitude slot may be relatively benign on a daily basis, the relative velocity - and combined risk factor - with which these near-misses occur may not be. Figure 4(b) illustrates worstcase GEO-relative velocities experienced in each $1^{\circ}$ longitude slot throughout the forecasting period. This relative velocity map demonstrates that even though a particular longitude slot may experience 1-2 near miss events per day at $50 \mathrm{~km}$, the maximum relative speed for these events could be upwards of $0.6 \mathrm{~km} / \mathrm{s}$ (approximately $1340 \mathrm{mph}^{12}$ ). In general, higher relative speeds can be attributed to uncontrolled objects with larger orbital inclinations, such that relative velocities at the ascending and descending nodes are increased with respect to

\footnotetext{
${ }^{11}$ The effect of nominal launch traffic on longitude-dependent congestion at GEO is simulated in Anderson and Schaub (2014).

${ }^{12}$ Relative velocities are lower in the GEO regime when compared to the LEO regime, since (a) orbit velocity is lower at the GEO altitude, and (b) objects at GEO are in general orbiting in the same direction (Hansen and Sorge, 2013). Compared to the 2009 Iridium-33/Cosmos-2251 collision, which occurred at a relative speed of upwards of $24,600 \mathrm{mph}(\sim 11 \mathrm{~km} / \mathrm{s})$ (Liou, 2009), $1340 \mathrm{mph}$ is a benign, but still mission-ending collision velocity, particularly if the debris impactor is large/massive (e.g., rocket body).
} 


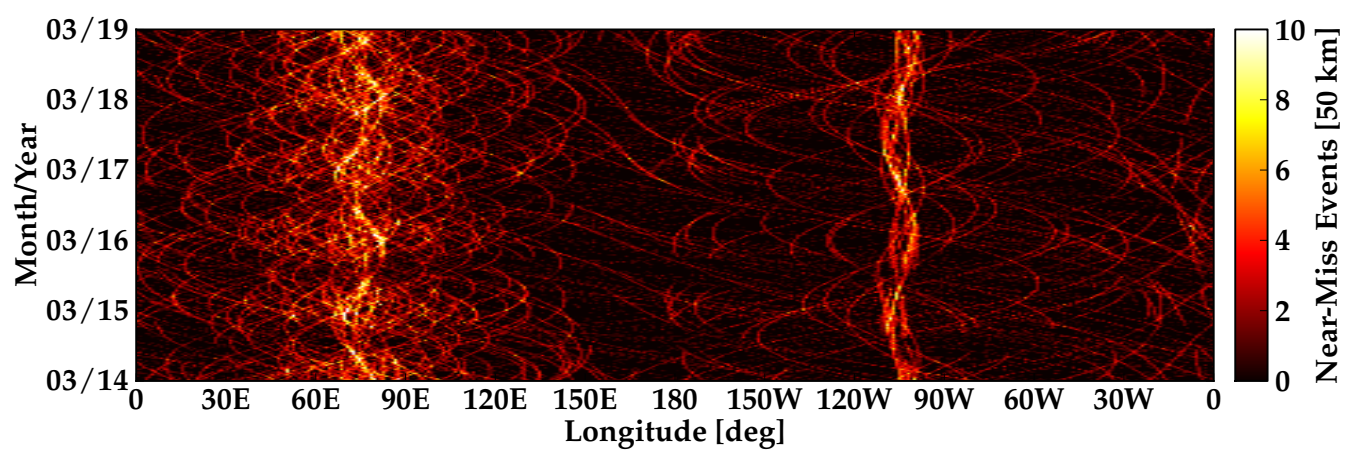

(a) Near-miss events per day at $50 \mathrm{~km}$ occurring during five-year forecasting period.

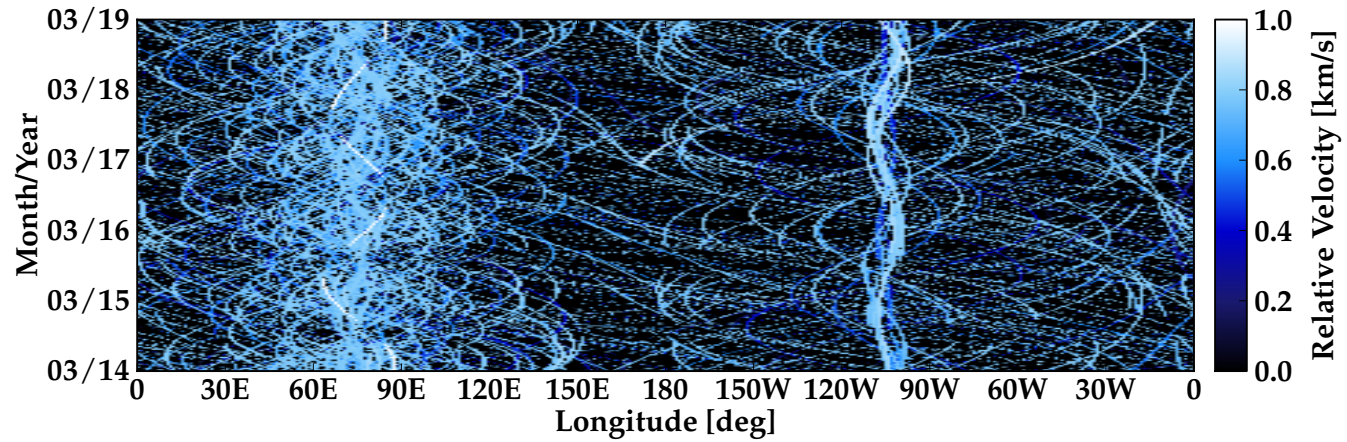

(b) Maximum relative velocities of near-miss events occurring during forecasting period.

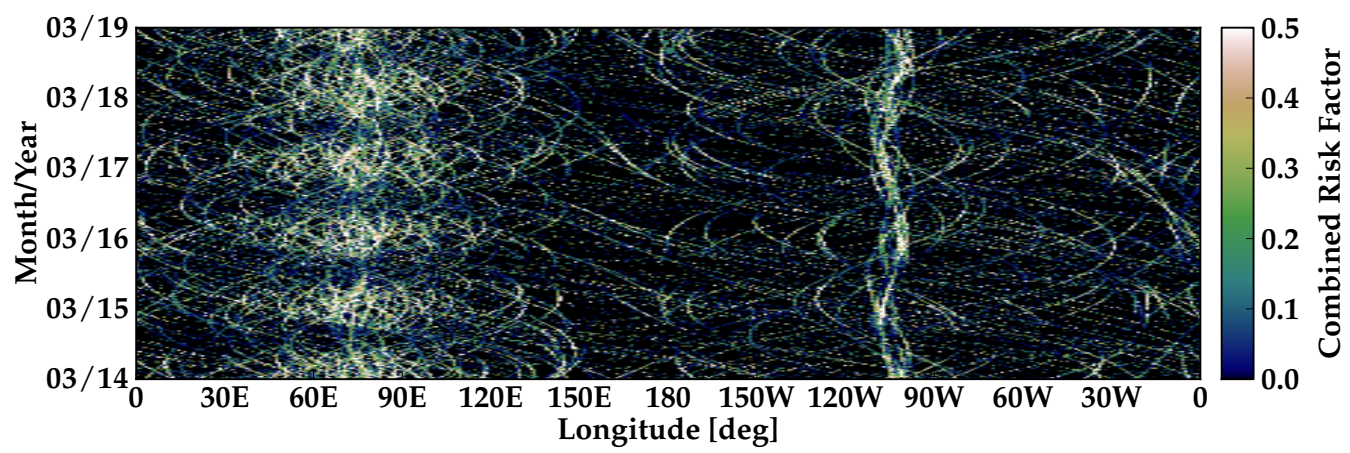

(c) Maximum combined risk factor of near-miss events occurring during forecasting period.

Figure 4: Three portraits of longitude-dependent congestion in the GEO ring arising from the large-scale, trackable, unclassified GEO debris population in the 02/28/2014 TLE set. 
equatorial GEO orbit velocity. The objects extracted from the TLE sets have nonzero eccentricity, however - each of these Keplerian elements serves to increase relative velocities at the nodes, rendering near-miss events with these particular objects more hazardous to operational assets.

Figure 4(c) illustrates worst-case combined risk factors experienced in each longitude slot throughout the forecasting period. Higher combined risk levels are visible in the vicinity of the gravitational wells, and exhibit a banding phenomenon driven by once-yearly oscillations in the magnitude of the eccentricity vector, induced by the SRP perturbation (Chao, 2005). Later in this paper, this combined risk map is used as one method of validation for an identified subset of high-risk objects, that is, the combined risk in the neighborhoods of the gravitational wells is attenuated if this high-risk subset of objects is omitted from the congestion forecast (representative of physical removal with an ADR technology).

In addition to tracking the number of near-miss events at $50 \mathrm{~km}$ occurring daily for each longitude slot over this five-year analysis period (cf. Figure 4), it is of interest to assess which classes of uncontrolled objects contribute the most to longitude-dependent congestion for purposes of recommending which types of objects to remove if a particular longitude slot is to be cleared of debris. Figure 5(a) shows the average number of near-miss events per day contributed by objects of each orbit class considered in this study. ${ }^{13}$ Longitude slots neighboring the debris-critical gravitational wells at $75^{\circ} \mathrm{E}$ and $105^{\circ} \mathrm{W}$ are subject to $3-4$ near-miss events per day, while less congested longitude slots experience only 0.5 near-miss events at $50 \mathrm{~km}$ per day on average. Congestion contributions in the regions surrounding the Eastern and Western gravitational wells are dominated by Eastern (L1) and Western (L2) librating objects, which oscillate in the Earth-fixed frame around these stable points with the libration periods shown in Figure 2. In addition, the drifting (D) objects contribute an approximate 0.25 near-misses per day across the entirety of the GEO ring, thereby generating the background noise observed in the debris congestion forecast in Figure 4(a). Interestingly, the near-miss contribution for the drift class peaks at the unstable equilibrium longitudes $165^{\circ} \mathrm{E}$ and $15^{\circ} \mathrm{W}$ - the longitudinal rate $\dot{\lambda}$ induced by the $J_{2,2}$ harmonic is at a minimum at these locations (Allan, 1963), causing drift class objects to linger longer at these longitudes (McKnight and Di Pentino, 2013).

In summary of the localized congestion, relative velocity, and combined risk results illustrated in Figure 4 for the five-year analysis period, Figures 5(b)-5(d) illustrate the average number of near-miss events at $50 \mathrm{~km}$ per day, beneath or above various relative position, relative velocity, and combined risk factor thresholds, respectively. As an example, the Eastern well at $75^{\circ} \mathrm{E}$ experiences an average of four near-misses per day at $50 \mathrm{~km}$ over the five-year forecast (Figure 5(a)), two of which are beneath $40 \mathrm{~km}$ in GEO proximity (Figure 5(b)) and greater than $0.75 \mathrm{~km} / \mathrm{s}$ in relative velocity (Figure 5(c)). Although Figure 5(c) illustrates that approximately all of the near-miss events occurring at $75^{\circ} \mathrm{E}$ are above the catastrophic velocity threshold in Equation (4), the combined risk summary in Figure 5(d) dictates that only one near-miss event per day at this longitude is above a combined risk level of 0.2. Therefore, the position risk function in Equation (3) is de-weighting the risk of these higher-

\footnotetext{
${ }^{13}$ The aggregation of near-miss events (or, equivalently, sub-50 km conjunctions) used in this average serves as a surrogate for slot-specific spatial densities contributed on average by members of each GEO orbit class.
} 
velocity encounters, since Figure 5(b) shows that the majority of these near-miss events are occurring above $25 \mathrm{~km}$ in GEO proximity, a distance beyond which the position risk function $R_{r}(r)$ is beneath 0.25 .

For purposes of slot safety assessment, it is useful to consider not only the mean of the number of near-misses occurring daily for each longitude slot, but the standard deviation for these distributions, as well. Figure 6 illustrates the $1 \sigma$ number of near-misses at $50 \mathrm{~km}$ for every longitude slot and risk factor threshold in Figure 5(d), highlighting the spread of these slot-specific distributions (generated by tracking the number of near-misses within each longitude slot, above each risk threshold, on each day over the five-year period). Nearmiss distributions around the two gravitational wells have a larger $1 \sigma$ spread for all risk thresholds, indicating that these congested regions can experience many more near-misses on a given day than the five-year average of approximately 3-4 near-misses per day shown in Figure 5(d). In particular, the near-miss distribution for $75^{\circ} \mathrm{E}$ extends from $0-13$ near-misses per day at $50 \mathrm{~km}$ in the analysis period, highlighting the insufficiency of the mean metric in Figure 5(d).

\subsection{Timing of Near-Miss Events}

In addition to the number of near-miss events per day for each GEO longitude slot, and the relative velocities and combined risk factors with which these near-miss events occur, it is beneficial to study the time of day at which these near-misses occur. McKnight and Di Pentino (2013) indicates that the equatorial crossing windows of the GEO debris population are synchronized such that collision hazard is episodic and predictable. To illustrate this concept, Figure 7 provides the distribution mean and 1/2/3 $\sigma$ corridors for the local time of all near-misses occurring across the GEO ring over each day of the first year of the forecasting period, for near-miss events during the ascending (south-to-north) equatorial pass in Figure 7(a), and descending (north-to-south) equatorial pass in Figure 7(b). The "tight" distributions shown in Figure 7 demonstrate that there exist two predictable windows relative to local noon during which observational coverage of GEO can be maximized, regardless of longitudinal location around the GEO ring. The equatorial crossing windows vary nearly linearly over the course of the year, and the ascending/descending windows occur $\sim 12$ hours apart from one another at any point in the year. These time-varying equatorial crossing windows are the Earth-fixed representation of the well-known GEO "pinch points," which are approximately static in inertial right ascension and declination space, and are leveraged for designing ground-based observation strategies and optimizing sensor tasking of space-based space surveillance (SBSS) systems (Sharma et al., 2002).

Relative to an Earth-fixed observer, the collective motion of the GEO debris population is similar to a transverse wave (McKnight and Di Pentino, 2013) - this motion arises from a clustering in right ascension of the ascending node, driven by luni-solar perturbations dominant at the GEO altitude. The equatorial crossing windows in Figure 7 are a physical manifestation of this phenomenon, and can be leveraged for maximizing observational coverage of the GEO ring, anomaly correlation, and forecasting of potential conjunction events. With this knowledge, the threat of near-miss events with higher combined risk factors can be mitigated through increased coverage of the desired slot during the predictable windows relative to local noon shown in Figure 7. 


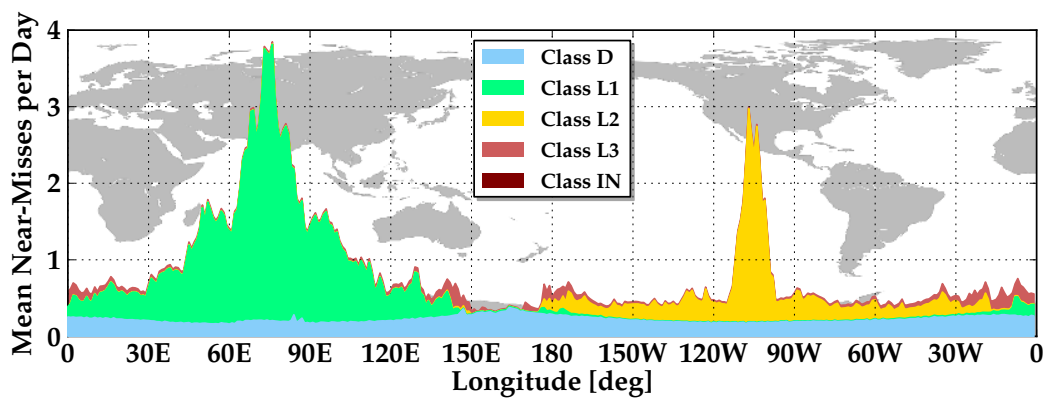

(a) Average near-miss events per day at $50 \mathrm{~km}$ by debris object class.

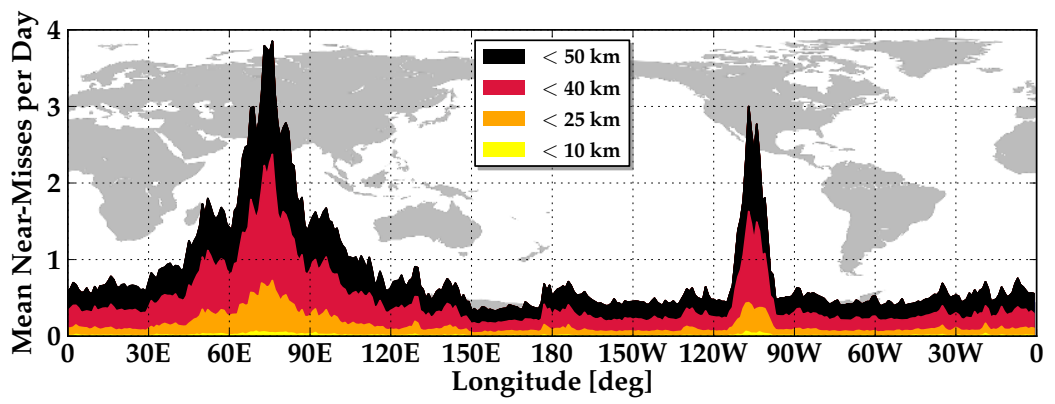

(b) Average near-miss events per day at $50 \mathrm{~km}$ by relative proximity.

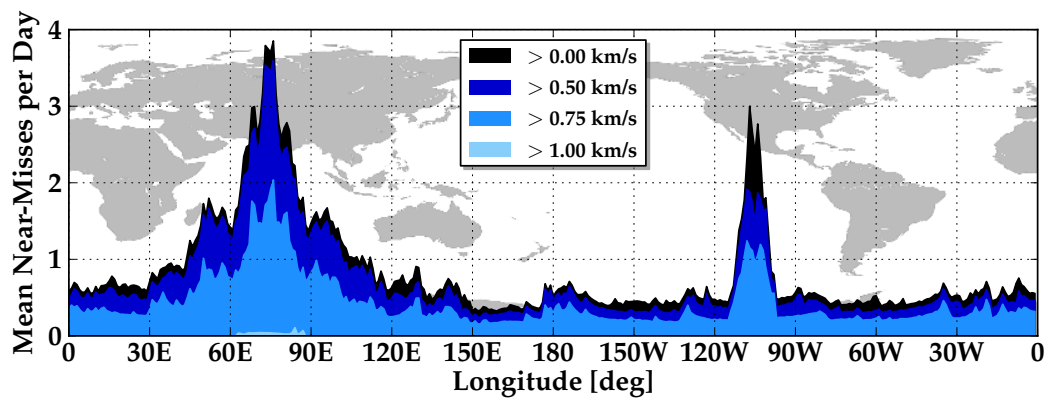

(c) Average near-miss events per day at $50 \mathrm{~km}$ by relative velocity.

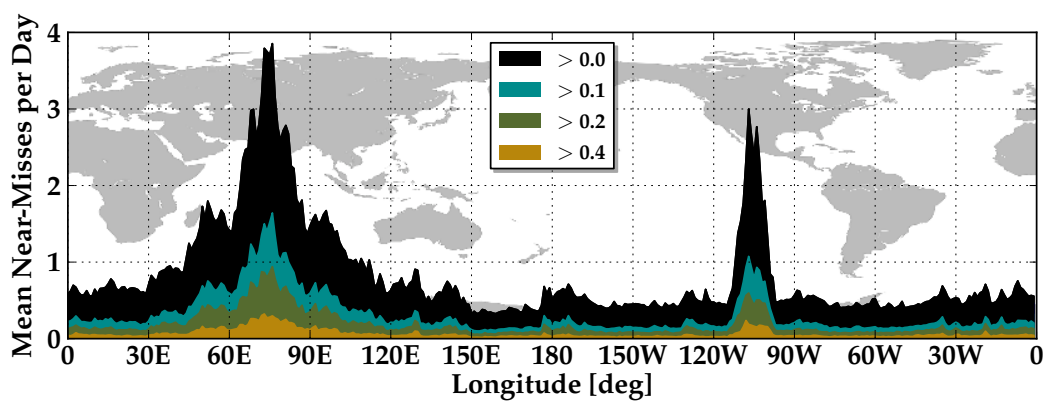

(d) Average near-miss events per day at $50 \mathrm{~km}$ by combined risk factor.

Figure 5: Average number of near-miss events per day for each longitude slot at GEO. 


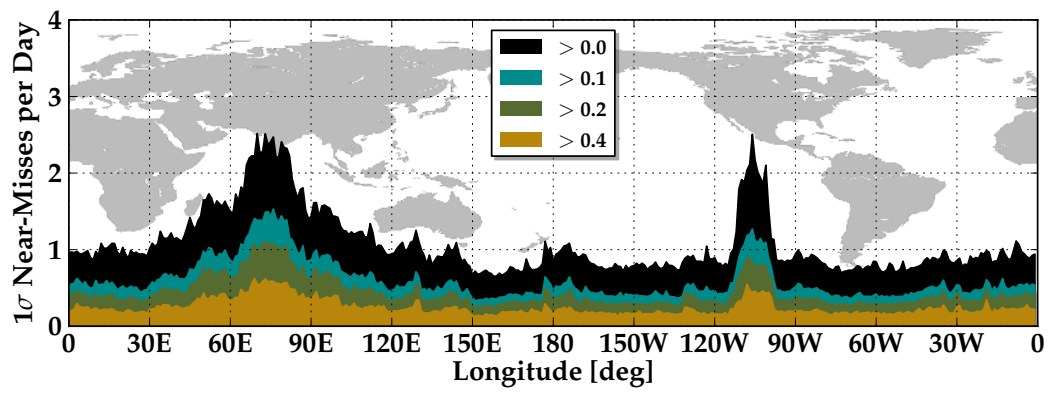

Figure 6: Standard deviation of near-miss events per day at $50 \mathrm{~km}$ by combined risk factor.

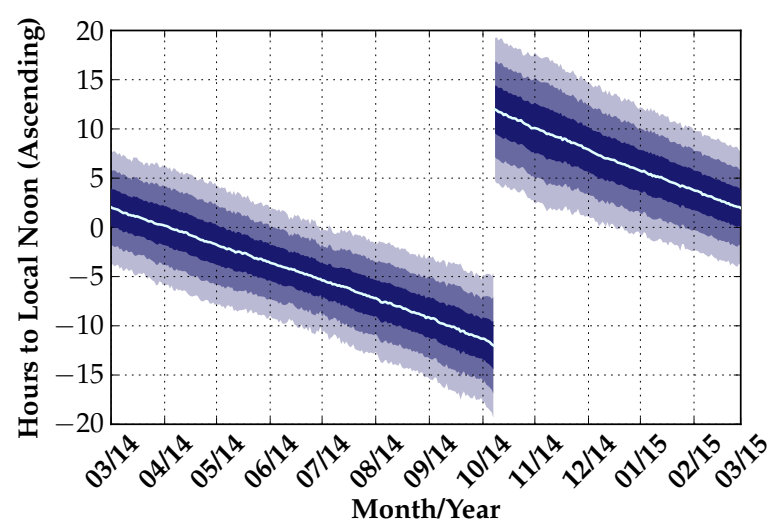

(a) Equator crossing time distribution (ascending).

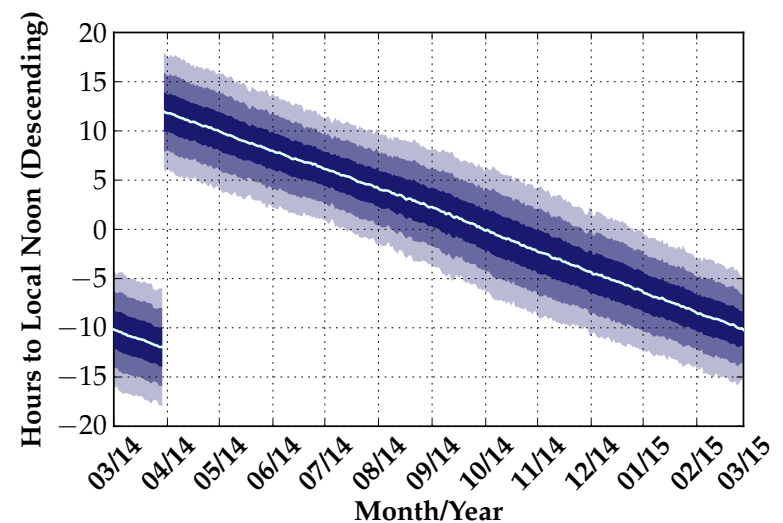

(b) Equator crossing time distribution (descending).

Figure 7: Equatorial crossing times for the GEO debris population are episodic and dependent upon time of year. Shaded swaths illustrate $1 / 2 / 3 \sigma$ corridors.

\section{Identifying Highest-Risk Debris Objects at GEO}

To begin identifying the classes of uncontrolled debris objects in the GEO regime that contribute the most to longitude-dependent congestion - and more importantly, longitudedependent risk - the results of the five-year congestion and risk forecast shown in Figure 4 are partitioned by debris class. Figure 8(a) gives a breakdown of the percentage that each uncontrolled orbit class contributed to the total number of near-miss events at $50 \mathrm{~km}$ occurring globally over the entire five-year analysis period. Figure 8(b) provides a breakdown of the percentage that each class contributed to the total combined risk of the near-miss events accumulated globally during the five-year analysis period. These pie charts are interesting when compared alongside the TLE orbit class breakdown in Figure 1. Although drift objects constitute $50.9 \%$ of the large-scale, trackable RSO population at GEO, they only contribute $26.3 \%$ of the near-miss events at $50 \mathrm{~km}$ detected during the forecasting period, which collectively account for $23.8 \%$ of the global risk accumulated during this period. Librating objects, on the other hand, contribute a dominating $73.1 \%$ of the number of near-misses - accounting for $76.1 \%$ of the global accumulated risk - even though these objects collectively make up a mere $14.1 \%$ of the RSO population at GEO (that is, $27.1 \%$ of the uncontrolled debris population in the 02/28/2014 TLE set). Of significant interest for ADR initiatives are the 105 


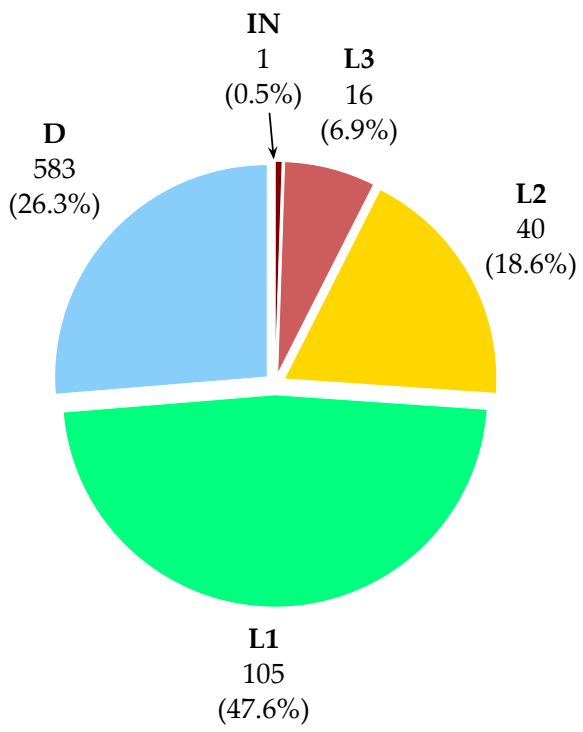

(a) Near-miss event contributions.

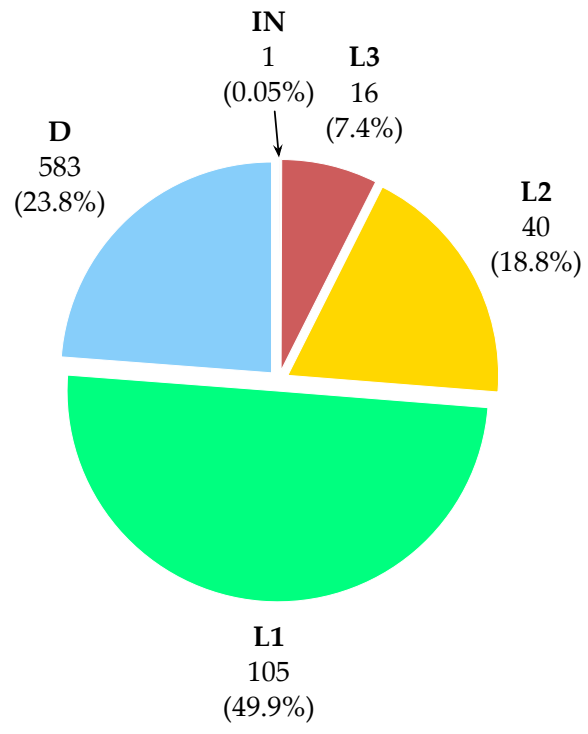

(b) Combined risk factor contributions.

Figure 8: Breakdown of globally contributed congestion and combined risk by orbit class.

Eastern librating (L1) derelicts that contribute $49.9 \%$ to the globally accumulated risk over the five-year forecast - that is, $14.1 \%$ of the uncontrolled debris population is collectively responsible for nearly $50 \%$ of global risk accumulated around GEO.

\subsection{Global Risk Accumulation Metric}

Although Figure 8 provides a congestion and risk breakdown by debris orbit class, it does not illustrate the contribution of individual objects within each orbit class to the combined risk globally accumulated during the five-year analysis period. One measure for identifying the highest-risk objects at GEO is the global risk accumulation metric, which utilizes the risk contribution of individual objects to the total risk globally accumulated around GEO during the forecasting period to rank the derelict population from highest to lowest risk level. To compute the global accumulated risk, the combined risk factors for all near-miss events occurring in all longitude slots throughout the five-year forecasting period are summed into a grand total. Then, the risk contributions of the near-miss events for individual objects to this grand total are applied to sort them into order of descending global risk contribution. Table 2 lists the top 10 highest-risk objects from the 02/28/2014 TLE set, identified using this global risk accumulation metric. Notably, all of these objects are defunct payloads that are from the L1/L2 librating classes. ${ }^{14}$ The semi-major axes for these high-risk objects are within \pm 15 $\mathrm{km}$ of the GEO radius at the beginning of the analysis period, and their inclinations are from $13-16^{\circ}$ relative to the equatorial plane. Collectively, these top 10 derelicts are responsible

\footnotetext{
${ }^{14}$ Since the first-order condition for stable point capture improves as proximity to the GEO semi-major axis decreases (Allan, 1963), this is an intuitive result. Librating objects have semi-major axes that are close to the synchronous radius, such that relative position risk in Equation (3) is higher, in general, for the near-miss events triggered by objects from these librating classes.
} 
for $9.39 \%$ of the total combined risk factor accumulated around the GEO ring throughout the five-year analysis period.

Table 2: Highest-risk debris objects in GEO regime, based on global risk accumulation (unweighted).

\begin{tabular}{c|l|c|c|c|c|c} 
Rank & Object Name & COSPAR & Class & Country & Slot Range & Contribution \\
\hline 1 & Aurora 1 & $1982-105 \mathrm{~A}$ & L2 & USA & $97-110^{\circ} \mathrm{W}$ & $0.968 \%$ \\
2 & Cosmos 1366 & $1982-044 \mathrm{~A}$ & L1 & USSR/Russia & $65-84^{\circ} \mathrm{E}$ & $0.965 \%$ \\
3 & ASC 1 & $1985-076 \mathrm{C}$ & L2 & USA & $79-130^{\circ} \mathrm{W}$ & $0.961 \%$ \\
4 & Raduga 1-2 & $1990-116 \mathrm{~A}$ & L1 & USSR/Russia & $49-100^{\circ} \mathrm{E}$ & $0.958 \%$ \\
5 & Insat 1C & $1988-063 \mathrm{~A}$ & L1 & India & $57-92^{\circ} \mathrm{E}$ & $0.949 \%$ \\
6 & GStar 1 & $1985-035 \mathrm{~A}$ & L2 & USA & $100-108^{\circ} \mathrm{W}$ & $0.947 \%$ \\
7 & GStar 3 & $1988-081 \mathrm{~A}$ & L2 & USA & $101-107^{\circ} \mathrm{W}$ & $0.931 \%$ \\
8 & Telstar 4A & $1993-077 \mathrm{~A}$ & L2 & USA & $97-113^{\circ} \mathrm{W}$ & $0.917 \%$ \\
9 & Raduga 26 & $1990-112 \mathrm{~A}$ & L1 & USSR/Russia & $55-94^{\circ} \mathrm{E}$ & $0.899 \%$ \\
10 & Cosmos 1897 & $1987-096 \mathrm{~A}$ & L1 & USSR/Russia & $49-100^{\circ} \mathrm{E}$ & $0.898 \%$ \\
\hline
\end{tabular}

It is critical to emphasize that this top 10 list is in effect for the next five years only, since it harnesses combined risk factor data for near-miss events detected during a five-year forecasting period. A well-known effect of luni-solar perturbations on GEO objects is longterm cyclical precession of the orbit plane, in which the inclination of initially equatorial objects will rise to $15^{\circ}$ and back with a period of approximately 53 years (Chao, 2005). Thus, in 26 years, the objects listed in Table 2 will be in nearly equatorial orbits, such that the relative speeds of these objects at the nodes is decreased, leading to diminished combined risk contributions for the near-miss events triggered by these currently high-risk derelicts.

Note that although the GEO-relative position and velocity at torus intersection are translated into a combined risk factor, the longitude at which the near-miss event occurs is not taken into account in the present risk formulation. Since operational GEO satellites are most often inserted into longitude slots in the neighborhood of the gravitational wells (Anderson and Schaub, 2014), it is useful to consider weighting the combined risk factor by the longitude of the near-miss event, to further emphasize higher-risk events occurring near these critical stable points, and de-weight higher-risk events occurring at longitudes with lower densities of operational satellites. An example of one such longitude weighting function is drawn from the wrapped-normal probability density function fit to past, present, and projected satellite longitude data in (Anderson and Schaub, 2014). This weighting function-illustrated in Figure 9-amplifies the combined risk experienced in longitude slots covering the United States and the Eurasian region, and reduces risk factors over the Atlantic and Pacific Oceans, where operational satellite densities are at a minimum.

Table 3 provides the top 10 highest-risk objects from the 02/28/2014 TLE set, identified using the global risk accumulation metric with longitudinal weighting applied. The first column provides the rank for each object under longitudinal weighting, and the number of places that these objects either rose or fell within the unweighted top 10 list in Table 2 as a result. The emphasis of the Eastern well over the Western well in Figure 9 serves to increase the ranking of Eastern librating (L1) objects in the ranked derelict population Aurora 1, the highest-risk L2 object from Table 2, falls three places in the weighted top 


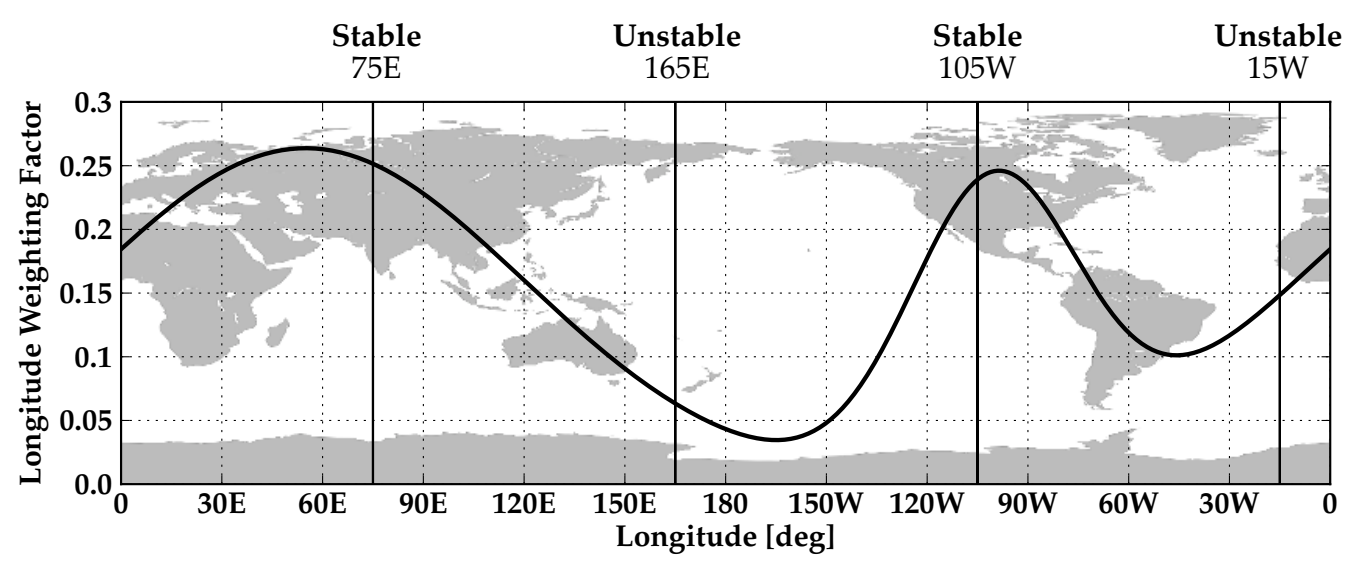

Figure 9: Longitude weighting function (from probability density function in (Anderson and Schaub, 2014)).

Table 3: Highest-risk debris objects in GEO regime, based on global risk accumulation (weighted).

\begin{tabular}{r|l|c|c|c|c|c} 
Rank & Object Name & COSPAR & Class & Country & Slot Range & Contribution \\
\hline $1(\uparrow 1)$ & Cosmos 1366 & $1982-044 \mathrm{~A}$ & L1 & USSR/Russia & $65-84^{\circ} \mathrm{E}$ & $1.226 \%$ \\
$2(\uparrow 3)$ & Insat 1C & $1988-063 \mathrm{~A}$ & $\mathrm{~L} 1$ & India & $57-92^{\circ} \mathrm{E}$ & $1.198 \%$ \\
$3(\uparrow 1)$ & Raduga 1-2 & $1990-116 \mathrm{~A}$ & $\mathrm{~L} 1$ & USSR/Russia & $49-100^{\circ} \mathrm{E}$ & $1.183 \%$ \\
$4(\downarrow 3)$ & Aurora 1 & $1982-105 \mathrm{~A}$ & $\mathrm{~L} 2$ & USA & $97-110^{\circ} \mathrm{W}$ & $1.168 \%$ \\
$5(\uparrow 1)$ & GStar 1 & $1985-035 \mathrm{~A}$ & L2 & USA & $100-108^{\circ} \mathrm{W}$ & $1.149 \%$ \\
$6(\uparrow 1)$ & GStar 3 & $1988-081 \mathrm{~A}$ & L2 & USA & $101-107^{\circ} \mathrm{W}$ & $1.131 \%$ \\
$7(\uparrow 2)$ & Raduga 26 & $1990-112 \mathrm{~A}$ & L1 & USSR/Russia & $55-94^{\circ} \mathrm{E}$ & $1.125 \%$ \\
$8(\uparrow 2)$ & Cosmos 1897 & $1987-096 \mathrm{~A}$ & L1 & USSR/Russia & $49-100^{\circ} \mathrm{E}$ & $1.117 \%$ \\
$9(\uparrow 3)$ & Cosmos 1961 & $1988-066 \mathrm{~A}$ & L1 & USSR/Russia & $68-82^{\circ} \mathrm{E}$ & $1.117 \%$ \\
$10(\uparrow 3)$ & Luch 1-1 & $1995-054 \mathrm{~A}$ & L1 & USSR/Russia & $72-77^{\circ} \mathrm{E}$ & $1.116 \%$ \\
\hline
\end{tabular}

10 list. Furthermore, two L2 objects from Table 2-ASC 1 and Telstar 4A-fall out of the top 10 completely when longitudinal weighting is applied. Collectively, the top 10 objects under longitudinal weighting are responsible for $11.53 \%$ of the total combined/weighted risk factor accumulated around GEO during the five-year analysis period, a higher percentage than that in Table 2.

To summarize the effect of the global risk accumulation metric in identifying the objects with the highest cumulative risk over the analysis period - that is, the derelicts that consistently come close to the GEO ring at consistently high relative velocities - Figure 10(a) provides contributed percent of global accumulated risk factor as a function of top percent of the ranked debris population, with and without longitudinal weighting. Recalling that the debris population is ranked in order of descending global risk contribution, Figure 10(a) shows that $60 \%$ of the derelicts (453 objects) do not contribute any risk to the accumulated total, i.e., $50 \mathrm{~km}$ near-miss events for these objects are not triggered during the five-year period. Without longitudinal weighting, the top 70 ranked objects are collectively responsible for $50 \%$ of global risk experienced during the forecasting period; under longitudinal weighting, this cumulative trend line becomes steeper, such that the top 59 objects are responsible 
for $50 \%$ of global accumulated risk, a result of increased risk contributions for the top ranked derelicts (cf. Tables 2-3).

\subsection{Local Risk Accumulation Metric}

If identification of the derelicts that contribute the highest accumulated risk to a particular subset of longitude slots is desired, the local risk accumulation metric can be evaluated in contrast to the global accumulation metric, which sums the combined risk for all near-miss events occurring across all longitude slots during the analysis period, local risk accumulation uses the sum of combined risk for only the near-miss events occurring in a subset of longitude slots during the analysis period. The local risk accumulation metric is useful for identifying objects that contribute the highest cumulative risk in the vicinity of the two debris-critical gravitational wells. Tables 4-5 show the top 10 highest-risk contributors to the total accumulated risk in $\pm 15^{\circ}$ longitude windows centered upon the Eastern and Western gravitational wells, respectively. Note that these 10 ranked objects in Table 4 contribute $29.94 \%$ of the combined risk accumulated around the Eastern well during the five-year analysis period, while these 10 ranked objects in Table 5 contribute $57.54 \%$ of the combined risk around the Western well accumulated during this period. This has significant implications for ADR initiatives seeking to attenuate local risk in the vicinity of the Western well: almost $60 \%$ of the combined risk accumulated in longitude slots in the interval $90-120^{\circ} \mathrm{W}$ is attributed to the top 10 objects in Table 5 alone. Note again that this risk measure is not associated with the probability of collision metric commonly used in conjunction assessment, and thus should not be treated as equivalent with this statistical measure.

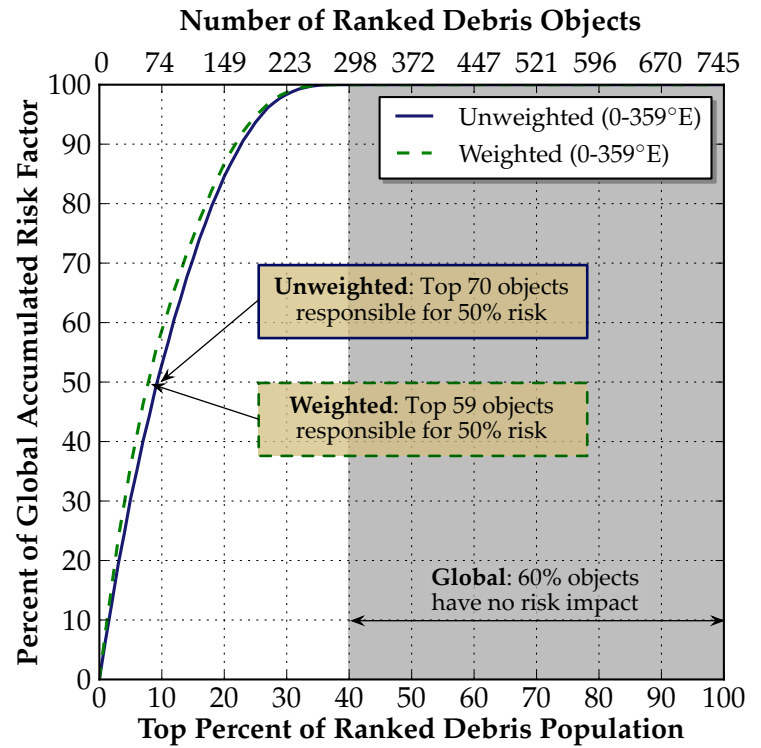

(a) Summary of global risk accumulated over GEO.

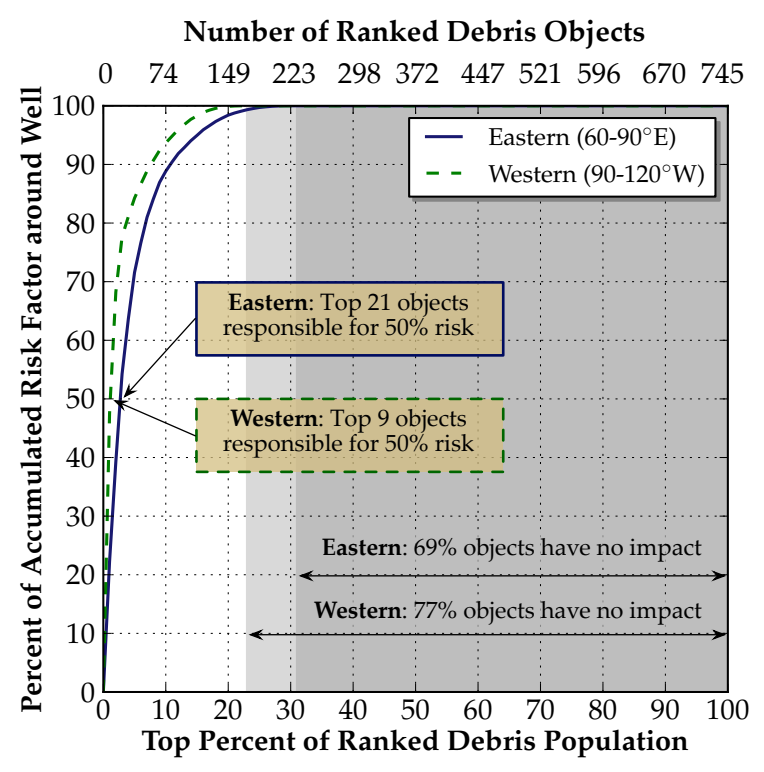

(b) Summary of local risk accumulated over wells.

Figure 10: Percent of accumulated risk factor as function of top percent of GEO debris population.

Figure 10(b) illustrates percent contributed to the accumulated risk around each well as a function of the top percent of the ranked debris population. From this chart, $69 \%$ 
Table 4: Highest-risk debris objects near Eastern well, based on local risk accumulation on $\left[60,90^{\circ} \mathrm{E}\right]$. Shaded rows indicate debris objects that are both globally high-risk (cf. Table 2) and locally high-risk.

\begin{tabular}{c|l|c|c|c|c|c} 
Rank & Object Name & COSPAR & Class & Country & Slot Range & Contribution \\
\hline 1 & Cosmos 1366 & $1982-044 \mathrm{~A}$ & L1 & USSR/Russia & $65-84^{\circ} \mathrm{E}$ & $3.636 \%$ \\
2 & Cosmos 1961 & 1988-066A & L1 & USSR/Russia & $68-82^{\circ} \mathrm{E}$ & $3.290 \%$ \\
3 & Luch 1-1 & 1995-054A & L1 & USSR/Russia & $72-77^{\circ} \mathrm{E}$ & $3.279 \%$ \\
4 & SIRIO 1 & $1977-080 \mathrm{~A}$ & L1 & Italy & $72-77^{\circ} \mathrm{E}$ & $3.115 \%$ \\
5 & Comstar 4 & $1981-018 \mathrm{~A}$ & L1 & USA & $69-80^{\circ} \mathrm{E}$ & $3.094 \%$ \\
6 & Cosmos 2133 & 1991-010A & L1 & USSR/Russia & $67-82^{\circ} \mathrm{E}$ & $2.971 \%$ \\
7 & Raduga 12 & 1983-028A & L1 & USSR/Russia & $63-85^{\circ} \mathrm{E}$ & $2.921 \%$ \\
8 & Raduga 14 & 1984-016A & L1 & USSR/Russia & $61-87^{\circ} \mathrm{E}$ & $2.637 \%$ \\
9 & Cosmos 2085 & $1990-061 \mathrm{~A}$ & L1 & USSR/Russia & $67-82^{\circ} \mathrm{E}$ & $2.504 \%$ \\
10 & Raduga 26 & $1990-112 \mathrm{~A}$ & L1 & USSR/Russia & $55-94^{\circ} \mathrm{E}$ & $2.490 \%$ \\
\hline \multicolumn{7}{l}{}
\end{tabular}

of derelicts (515 objects) do not contribute to accumulated Eastern well risk, and $77 \%$ of derelicts (574 objects) do not contribute to accumulated Western well risk. As a result, these cumulative trend lines for the local accumulated risk in the vicinity of the gravitational wells in Figure 10(b) are steeper than those for the global accumulated risk in Figure 10(a). In particular, the top 21 highest-risk derelicts near the Eastern well - and the top 9 highest-risk objects near the Western well (cf. Table 5) - are responsible for 50\% of the localized risk observed in the $30^{\circ}$ longitude regions centered on each gravitational well, respectively.

To illustrate how removal of the highest cumulative risk objects in Tables 4-5 attenuates localized risk in the vicinity of the gravitational wells throughout the five-year analysis period, Figure 11 shows the mean number of near-miss events per day at $50 \mathrm{~km}$ above various combined risk levels, with the full GEO debris population from the 02/28/2014 TLE data set (Figure 5(d)), with the top 5 derelicts in Tables 4-5 removed (Figure 11(a)), and with all 10 derelicts in Tables 4-5 removed (Figure 11(b)). As Figure 11 shows, removal of the highest cumulative risk objects in the vicinity of the gravitational wells serves to attenuate localized risk in these critical regions. In particular, Figure 11(b) illustrates that removal of the 10 locally high-risk objects identified in Table 5 not only leads to a $50 \%$ reduction in the mean number of near-miss events occurring around the Western well, but nearly eliminates any near-miss events with combined risk factors greater than 0.4 during the five-year period. Again, since debris-debris collision risk is not considered in this analysis, this $50 \%$ reduction in localized congestion via the near-miss event metric should be interpreted as a reduction in potential collision risk to operational satellites from large-scale derelicts at GEO only, not as a reduction in total risk associated with particular debris objects colliding with one another.

\subsection{Absolute Worst-Case Risk Metric}

The global and local risk accumulation metrics use the sum of the combined risk factors for near-miss events detected across all longitude slots - or a defined subset thereof - throughout the analysis period. Although these two measures are useful for identifying the GEO debris objects responsible for the highest levels of cumulative risk experienced either globally or locally at GEO, these metrics do not guarantee that the derelicts responsible for the highest 
Table 5: Highest-risk debris objects by Western well, based on local risk accumulation on $\left[90,120^{\circ} \mathrm{W}\right]$. Shaded rows indicate debris objects that are both globally high-risk (cf. Table 2) and locally high-risk.

\begin{tabular}{c|l|c|c|c|c|c} 
Rank & Object Name & COSPAR & Class & Country & Slot Range & Contribution \\
\hline 1 & Aurora 1 & $1982-105 \mathrm{~A}$ & L2 & USA & $97-110^{\circ} \mathrm{W}$ & $8.215 \%$ \\
2 & GStar 1 & $1985-035 \mathrm{~A}$ & L2 & USA & $100-108^{\circ} \mathrm{W}$ & $8.037 \%$ \\
3 & GStar 3 & $1988-081 \mathrm{~A}$ & L2 & USA & $101-107^{\circ} \mathrm{W}$ & $7.902 \%$ \\
4 & Telstar 4A & $1993-077 \mathrm{~A}$ & L2 & USA & $97-113^{\circ} \mathrm{W}$ & $7.780 \%$ \\
5 & GOES 3 & $1978-062 \mathrm{~A}$ & L2 & USA & $99-109^{\circ} \mathrm{W}$ & $5.449 \%$ \\
6 & LES 8 & $1976-023 \mathrm{~A}$ & L2 & USA & $97-111^{\circ} \mathrm{W}$ & $5.364 \%$ \\
7 & LES 9 & $1976-023 \mathrm{~B}$ & L2 & USA & $99-111^{\circ} \mathrm{W}$ & $4.821 \%$ \\
8 & ASC 1 & $1985-076 \mathrm{C}$ & L2 & USA & $79-130^{\circ} \mathrm{W}$ & $3.630 \%$ \\
9 & NATO IIB & $1971-009 A$ & L2 & USA & $102-108^{\circ} \mathrm{W}$ & $3.218 \%$ \\
10 & Solidaridad 1 & $1993-073 \mathrm{~A}$ & L2 & Mexico & $100-111^{\circ} \mathrm{W}$ & $3.124 \%$ \\
\hline
\end{tabular}

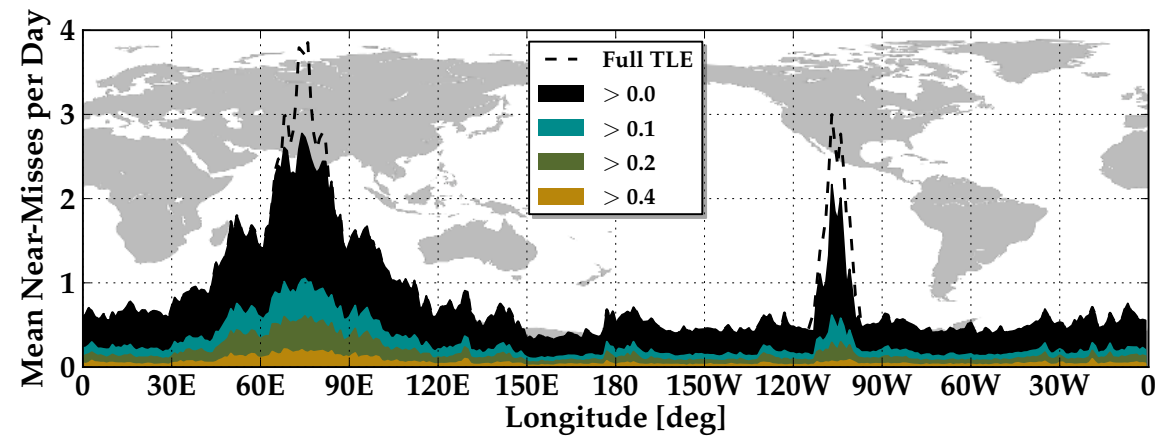

(a) Average number of near-misses by combined risk factor (top 5 removed).

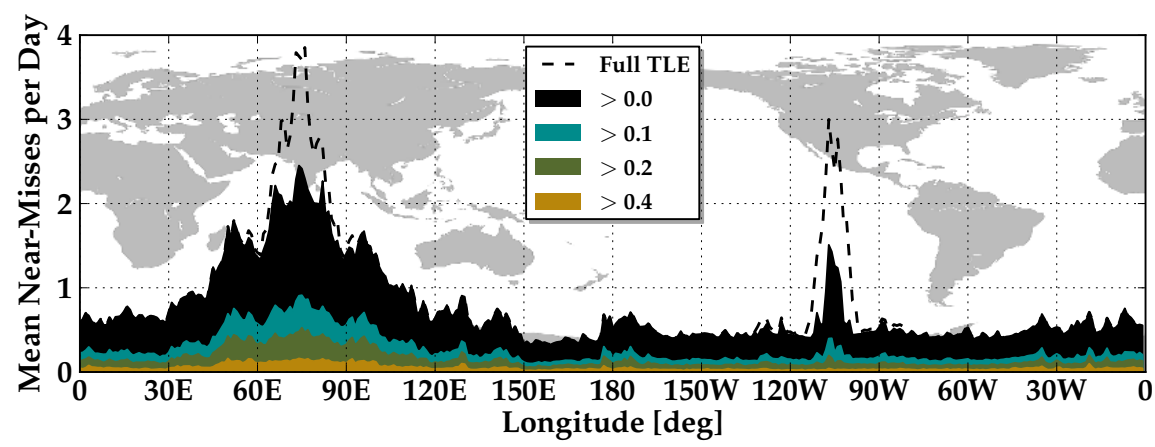

(b) Average number of near-misses by combined risk factor (top 10 removed).

Figure 11: Removal of highest-risk debris objects (Tables 4-5) from 02/28/2014 TLE attenuates local risk for operational assets stationed near the gravitational wells.

absolute risk near-miss events will be identified in the ranking scheme. Thus, instead of ranking the debris population by risk contribution to a globally or locally accumulated risk total, the absolute worst-case risk metric ranks these debris objects by the maximum singleevent risk factor each object contributed during the analysis period. 
Table 6 compares the top 5 globally highest-risk objects, identified via (a) the global accumulation metric (cf. Table 2), and (b) the absolute worst-case metric. Although these risk contributions for the accumulated risk subset are the highest of the population, the worst-case risk events for these objects are not as severe as the worst-case events for the absolute risk subset. Conversely, the absolute risk subset captures the highest single-event risks observed during the analysis period, but the cumulative risk contributions for these objects are far beneath the contributions of the accumulated risk subset - this is largely a consequence of the number of near-miss events that each derelict is responsible for during the analysis period (compare the number of near-misses for the accumulated risk subset with the number of near-misses for the absolute risk subset in Table 6).

As the longitude interval used for risk accumulation decreases in length, the percent contribution and worst risk differences between the accumulated risk and absolute risk subsets are amplified, i.e., the worst single-event risk for the highest accumulated risk object in a single longitude slot could be significantly smaller than the worst single-event risk in that slot for the highest absolute risk object. Therefore, depending upon the objectives for a particular ADR initiative - that is, whether the effort is geared towards reducing cumulative or absolute risk in a particular subset of longitude slots - two different methodologies are presented to identify the highest-risk target objects for either objective.

\begin{tabular}{|c|c|c|c|c|c|c|c|}
\hline & Rank & Object Name & COSPAR & Class & Events & Contribution & Worst Risk \\
\hline \multirow{5}{*}{ 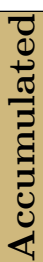 } & 1 & Aurora 1 & $1982-105 \mathrm{~A}$ & L2 & 3666 & $0.968 \%$ & 0.886 \\
\hline & 2 & Cosmos 1366 & $1982-044 \mathrm{~A}$ & L1 & 3668 & $0.965 \%$ & 0.952 \\
\hline & 3 & $\mathrm{ASC} 1$ & 1985-076C & L2 & 3593 & $0.961 \%$ & 0.929 \\
\hline & 4 & Raduga 1-2 & 1990-116A & L1 & 3400 & $0.958 \%$ & 0.911 \\
\hline & 5 & Insat IC & $1988-063 \mathrm{~A}$ & L1 & 3568 & $0.949 \%$ & 0.884 \\
\hline \multirow{5}{*}{ 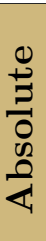 } & $\overline{1} 1$ & Syncom 2 & $\overline{1963-031 \mathrm{~A}}$ & $\overline{\mathrm{D}}$ & 1753 & $0.438 \%$ & 0.993 \\
\hline & 2 & IUS stage 2 & 1991-054D & L3 & 1293 & $0.264 \%$ & 0.990 \\
\hline & 3 & LES 9 & 1976-023B & L2 & 2443 & $0.568 \%$ & 0.981 \\
\hline & 4 & Raduga 7 & $1980-081 \mathrm{~A}$ & L2 & 1960 & $0.311 \%$ & 0.980 \\
\hline & 5 & Satcom 3R & 1981-114A & $\mathrm{D}$ & 2121 & $0.511 \%$ & 0.979 \\
\hline
\end{tabular}

\section{Conclusions}

In this study, forecasting of localized debris congestion in the GEO ring is performed to determine how many near-miss events at $50 \mathrm{~km}$ occur on a daily basis for each of the GEO longitude slots over a five-year analysis period. The GEO-relative position and velocity at torus intersection for each near-miss event are translated into a combined risk factor that gauges how threatening the near-miss is for a hypothetical GEO satellite stationed at the longitude of intersection. Three methods for identifying the highest-risk debris objects at GEO are presented to provide a framework for determining the top ADR targets at GEO. Two methods use the contribution to a combined risk total accumulated either globally or over a subset of longitude slots to rank the debris population from highest to lowest risk; 
the third method considers the maximum single-event risk as the measure for ranking the population.

Simulation results dictate that the librating class objects - especially those of the Eastern librating class - are the most responsible for both global and localized risk levels, especially in longitude slots surrounding the two gravitational wells at $75^{\circ} \mathrm{E}$ and $105^{\circ} \mathrm{W}$. In the vicinity of the Eastern well, the top 21 ranked objects account for $50 \%$ of the accumulated risk in this region over the five-year analysis period, and in the vicinity of the Western well, the top 9 ranked objects are responsible for $50 \%$ of the accumulated risk in this region. Therefore, the results of this risk study have significant implications for ADR initiatives aimed at attenuating orbit debris risk, either globally or locally, in the GEO ring. Removal of the top 10 highest cumulative risk objects near the Western well leads to a $50 \%$ reduction in the average number of near-miss events occurring in this region, and nearly eliminates all near-miss events in this region occurring with significant risk factors, that is, those that could potentially warrant evasive action by operators. Ultimately, this study serves to begin a dialogue concerned with identifying the highest-risk debris objects at GEO for purposes of ADR target selection in this arena.

\section{Acknowledgment}

The authors would like to acknowledge the U.S. Department of Defense and the National Defense Science and Engineering Graduate Fellowship (NDSEG), the program through which funding for this research was obtained. The authors furthermore acknowledge Dr. Brandon Jones and the TurboProp software suite (Hill and Jones, 2009), from which the integration routines employed in this research were obtained. Finally, the authors would like to thank the anonymous reviewers who strengthened the content of this manuscript.

Allan, R. R., August 1963. Perturbations of a Geostationary Satellite by the LongitudeDependent Terms in the Earth's Gravitational Field. Planetary and Space Science 11, $1325-1334$.

Anderson, P. V., Schaub, H., June 2013. Local Orbital Debris Flux Study in the Geostationary Ring. Advances in Space Research 51 (12), 2195-2206.

Anderson, P. V., Schaub, H., February 2014. Local debris congestion in the geosynchronous environment with population augmentation. Acta Astronautica 94 (2), 619-628.

Berry, M. M., Healy, L. M., July-September 2004. Implementation of gauss-jackson integration for orbit propagation. Journal of the Astronautical Sciences 52 (3), 331-357.

Chao, C.-C., 2005. Applied Orbit Perturbation and Maintenance. The Aerospace Press.

Chobotov, V. A., 2002. Orbital Mechanics. American Institute of Aeronautics and Astronautics, Inc.

Chrystal, P., McKnight, D., Meredith, P., 2011. Space debris: On collision course for insurers? Tech. rep., Swiss Reinsurance Company Ltd. 
Curtis, H., 2005. Orbital Mechanics for Engineering Students. Elsevier ButterworthHeinemann.

Flegel, S., Gelhaus, J., Wiedemann, C., Vrsmann, P., Oswald, M., Stabroth, S., Klinkrad, H., Krag, H., 2009. The MASTER-2009 Space Debris Environment Model. In: Proceedings of the Fifth European Conference on Space Debris. No. ESA SP-672.

Flohrer, T., February 2014. Classification of Geosynchronous Objects: Issue 16. Tech. Rep. 1, European Space Operations Centre.

Flohrer, T., Lemmens, S., Virgili, B. B., Krag, H., Klinkrad, H., Parrilla, E., Sanchez, N., Oliveira, J., Pina, F., 2013. DISCOS - Current Status and Future Developments. In: Proceedings of the Sixth European Conference on Space Debris. No. ESA SP-723.

Hanada, T., Yasaka, T., Hata, H., Akahoshi, Y., September-October 2005. Using NASA Standard Breakup Model to Describe Low-Velocity Impacts on Spacecraft. Journal of Spacecraft and Rockets 42 (5).

Hansen, B. W., Sorge, M. E., August 2013. Summarizing the general effects of breakup debris in geo. In: Proceedings of the 2013 AAS/AIAA Astrodynamics Specialist Conference. No. 13-844.

Hill, K., Jones, B. A., May 2009. TurboProp Version 4.0. Colorado Center for Astrodynamics Research, University of Colorado at Boulder.

Hoots, F. R., Roehrich, R. L., December 1980. Spacetrack report no. 3: Models for propagation of norad element sets. Tech. rep., Office of Astrodynamics, Aerospace Defense Center.

Jehn, R., Agapov, V., Hernandez, C., 2005. The situation in the geostationary ring. Advances in Space Research 35, 1318-1327.

Johnson, N., 1999. Protecting the geo environment: Policies and practices. Space Policy 15, $127-135$.

Johnson, N. L., Krisko, P. H., Liou, J.-C., Anz-Meador, P. D., 2001. Nasa's new breakup model of evolve 4.0. Advances in Space Research 28 (9), 1377-1384.

Klinkrad, H., 2006. Space Debris: Models and Risk Analysis. Praxis Publishing.

Lewis, H., Swinerd, G., Williams, N., Gittins, G., March 2001. Damage: A dedicated geo debris model framework. In: Proceedings of the Third European Conference on Space Debris. Vol. 1.

Liou, J.-C., April 2009. Satellite collision leaves significant debris clouds. In: Orbital Debris Quarterly News. Vol. 13. NASA Orbital Debris Program Office, pp. 1-2.

Liou, J.-C., 2011. An active debris removal parametric study for leo environmental remediation. Advances in Space Research 47, 1865-1876. 
Luu, K., Sabol, C., October 1998. Effects of perturbations on space debris in supersynchronous storage orbits. Tech. rep., Air Force Research Laboratory.

McKnight, D. S., July-August 1991. Determination of Breakup Initial Conditions. Journal of Spacecraft and Rockets 28 (4), 470-477.

McKnight, D. S., Di Pentino, F. R., 2013. New Insights on the Orbital Debris Collision Hazard at GEO. Acta Astronautica 85, 73-82.

Montenbruck, O., Gill, E., 2000. Satellite Orbits: Models, Methods, Applications. Springer.

Oltrogge, D., Finkleman, D., August 2008. Consequences of debris events in geosynchronous orbit. In: Proceedings of the 2008 AIAA/AAS Astrodynamics Specialist Conference and Exhibit. No. 2008-7375.

Peterson, G. E., 2012. Target identification and delta-v sizing for active debris removal and improved tracking campaigns. In: Proceedings of the International Symposium on Space Flight Dynamics.

Quinlan, J. R., Jones, C. A., Vittaldev, V., Wilhite, A., September 2011. On the design of an active debris removal architecture for low earth orbit space debris remediation. In: Proceedings of the 2011 AIAA SPACE Conference and Exposition.

Schaub, H., Jasper, L., August 2011. Circular orbit radius control using electrostatic actuation for 2-craft configurations. In: Proceedings of the 2011 AAS/AIAA Astrodynamics Specialist Conference.

Sharma, J., Stokes, G. H., von Braun, C., Zollinger, G., Wiseman, A. J., 2002. Toward Operational Space-Based Space Surveillance. Lincoln Laboratory Journal 13 (2), 309-334.

USNO, UKHO, 2013. The Astronomical Almanac for the Year 2013. U. S. Nautical Almanac Office with Her Majesty's Nautical Almanac Office.

Vallado, D., 2007. Fundamentals of Astrodynamics and Applications, 3rd Edition. Microcosm Press.

Vallado, D. A., Crawford, P., Hujsak, R., Kelso, T. S., August 2006. Revisiting spacetrack report no. 3: Revision 2. In: Proceedings of the 2006 AIAA/AAS Astrodynamics Specialist Conference.

Wegener, P., Bendisch, J., Krag, H., Oswald, M., Stabroth, S., 2004. Population evolution in the geo vicinity. Advances in Space Research 34, 1171-1176. 